\title{
La investigación en Tecnología Educativa y las nuevas ecologías del aprendizaje: Design-Based Research (DBR) como enfoque metodológico.
}

\section{Research in Educational Technology and new ecologies of learning: Design-Based Research (DBR) as a methodological approach.}

\author{
Jesús Valverde-Berrocoso \\ Universidad de Extremadura \\ jevabe@unex.es
}

Recibido: 01/05/2016

Aceptado: $10 / 05 / 2016$

Publicado: 30/06/2016

\section{RESUMEN}

La investigación en Tecnología Educativa ha experimentado un amplio crecimiento en las últimas décadas. Este desarrollo no ha tenido una influencia significativa en la práctica educativa. La integración de las tecnologías digitales en los sistemas educativos no ha modificado sustancialmente la metodología docente para adaptarse a las competencias del siglo XXI. La investigación educativa tiene un escaso impacto en la toma de decisiones de los profesionales de la educación. Por otra parte, las nuevas ecologías del aprendizaje nos sitúan ante contextos de enseñanza-aprendizaje más flexibles, abiertos, distribuidos y conectados. Se necesitan nuevas teorías del conocimiento y nuevos enfoques en las metodologías de investigación educativa. En este artículo el «aprendizaje trialógico» se identifica como un marco conceptual útil para la comprensión de los nuevos contextos educativos y el «Design-Based Research» (DBR) se describe como un modelo metodológico idóneo para la integración efectiva de la investigación en la teoría y práctica pedagógica.

\section{PALABRAS CLAVE}

Tecnología Educativa; Ecologías de Aprendizaje; Aprendizaje Trialógico; Metodología de investigación; Investigación basada en Diseño.

\section{ABSTRACT}

The research in Educational Technology has experienced extensive growth in recent decades. This development has not had a significant influence on educational practice. The integration of digital technologies in educational systems has not substantially changed the teaching methodology to adapt the 21st Century Competences. Educational research has little impact on decision-making of education professionals. Moreover, new learning ecologies put us in contexts of teaching and learning more flexible, open, distributed and connected. New theories of knowledge and new approaches in educational research methodologies are needed. In this article the "trialogical learning» is identified as a useful conceptual framework for understanding the new educational contexts and "Design-Based Research" (DBR) is described as a suitable methodological model for the effective integration of research in the pedagogical theory and practice.

\section{KEYWORDS}

Educational Technology; Learning Ecologies; Trialogical Learning; Research Methodology; Design-Based Research.

\section{CITA RECOMENDADA}

Valverde-Berrocoso, J. (2016). La investigación en Tecnología Educativa y las nuevas ecologías del aprendizaje: Design-Based Research (DBR) como enfoque metodológico. RIITE. Revista Interuniversitaria de Investigación en Tecnología Educativa, 0, 60-73. Doi: http://dx.doi.org/10.6018/riite/2016/257931 


\section{INTRODUCCIÓN}

La investigación en Tecnología Educativa (en adelante TE) en las últimas décadas ha generado una importante producción que se ha visto reflejada en un crecimiento significativo de artículos en revistas científicas especializadas y no especializadas en este ámbito. Recientemente se han realizado estudios de revisión de la investigación en TE que nos ofrecen una visión global acerca de los temas y las tendencias sobre la Educación Digital (v.gr. Baydas, Kucuk, Yilmaz, Aydemir, y Goktas, 2015; Hsu, Hung, y Ching, 2013). El estudio de Baydas et al. (2015) analizó los temas y métodos de investigación en artículos sobre TE publicados durante el período 2002-2014 ( $\mathrm{N}=1255)$ en las prestigiosas revistas British Journal of Educational Technology (BJET) y Educational Technology Research and Development (ETRyD). Como resultado del estudio se identificaron diferentes temas de investigación agrupados en las siguientes categorías:

- Teorías/Enfoques de aprendizaje: aprendizaje cognitivo (estilos de aprendizaje/cognitivos, mapas conceptuales, aprendizaje autorregulado), modelos mentales, aprendizaje colaborativo, aprendizaje basado en solución de problemas, aprendizaje asistido por ordenador, mobile learning, constructivismo, aprendizaje personalizado, teoría de la carga cognitiva, blended learning, comunidad de aprendizaje, aprendizaje basado en casos.

- Entornos de aprendizaje: multimedia/hipermedia, videojuegos educativos, herramientas web, mundos virtuales, software educativo (desarrollo de software, agentes pedagógicos, sistemas de apoyo al desempeño), objetos de aprendizaje, web 2.0, simulaciones, web 3.0 o web semántica.

- Investigación en tecnología educativa: integración TIC, aprendizaje interactivo, análisis de contenido, modelo de Aceptación, Políticas, Programas.

- Aprendizaje online: e-Learning, comunicación online (debates), LMS (Sistemas de gestión de aprendizaje)

- Estudios de evaluación: evaluación de aprendizajes, evaluación de programas, feedback, e-portfolio.

- Diseño instruccional: diseño curricular, diseño de juegos.

Además de identificar los temas de investigación en TE, también comprobaron que los estudios cuantitativos son los más frecuentes $(36 \%)$ seguidos por los cualitativos $(27 \%)$. Los estudios cuasi-experimentales son los más utilizados entre los métodos cuantitativos y los estudios de caso son los más usados entre los métodos cualitativos. Las técnicas de recogida de datos más habituales son los cuestionarios (estudios cuantitativos), documentos y entrevistas (estudios cualitativos). Como conclusiones del estudio, Baydas et al. (2015) recomiendan ampliar la investigación relacionada con las políticas educativas TIC dada su relevancia para la integración de las tecnologías digitales en los sistemas educativos. También sugieren el uso de métodos mixtos que faciliten una mayor integración teoría-práctica en el campo de estudio de la TE. Asimismo consideran necesario el uso de técnicas alternativas de evaluación para enriquecer los resultados. Con respecto a las muestras creen relevante que se incluya en las investigaciones a familias y gestores educativos. Concluyen llamando la atención sobre las tendencias metodológicas que marcan las líneas editoriales de las revistas analizadas y que, por tanto, influyen en el proceso de selección de los artículos.

Por otra parte, Hsu et al. (2013) llevaron a cabo una revisión de la investigación en TE como concepto global durante el período de 2000-1010, a partir de los artículos publicados en seis revistas especializadas en este ámbito, todas ellas incluidas en el SSCI (Social Science Citation Index) y situadas entre las 50 primeras: British Journal of Educational Technology (BJET), Computers y Education (CyE), Journal of Educational Technology and Society (JETS), Educational Technology Research y Development (ETRyD), Innovations in Education and Teaching International (IETI) y Journal of Computer Assisted Learning (JCAL). Estas revistas tienen en común, además, que utilizan la revisión por pares, centran sus contenidos en la aplicación de las tecnologías con fines educativos, tienen una elevada reputación en el ámbito de la TE y poseen un carácter internacional. El número de artículos publicados por años revela un crecimiento total cercano al $20 \%$, siendo la revista Computers y Education la que muestra un mayor incremento de artículos en los últimos cuatro años. En el año 2000 las seis revistas publicaron unos 100 artículos y en 2010 la cifra está cerca de los 500 artículos. La longitud 
media de los artículos está entre las 10-15 páginas. La revista con mayor extensión en sus artículos es ETRyD y la de menor extensión CyE. Por país de origen de los autores, las revistas BJET e IETI son mayoritariamente del Reino Unido, en ETRyD de los Estados Unidos, en las publicaciones JETS, CyE y JCAL predominan autores de Taiwan y Estados Unidos. Los 10 países con mayor número de publicaciones en el ámbito de la Tecnología Educativa son (en orden de mayor a menor): USA, Reino Unido, Taiwan, Australia, Holanda, Canadá, Turquía, Grecia, Singapur y Alemania.

Para esta revisión se utilizó la técnica «Text Mining» que permite analizar información de grandes colecciones de textos y obtener patrones y relaciones en los datos de dichos documentos. Tras el análisis se identificaron 19 clusters de los que se extrajeron tres grandes dimensiones y 6 grupos. La figura 1 recoge la estructura jerárquica resultante.

Se comprobó que los temas con más de 15 artículos publicados por año fueron los siguientes: Macro-visión del Aprendizaje On-line (45); Aprendizaje interactivo y aprendizaje colaborativo on-line (37); Macro-visión de la Integración de Tecnologías (25); Diseño instruccional (23); Software educativo y simulación (23); Evaluación en entornos basados en tecnología (17); Sistemas instructivos automatizados (17). Por otra parte, los temas más estables, en media de artículos por año, a lo largo del período 2000-2010 fueron: Aprendizaje basado en tecnología (15); Actitudes hacia la tecnología (11); Comunidad de aprendizaje (9); Debates on-line (8); Solución de problemas (7) y Juegos educativos (7). Y los temas menos desarrollados (promedio de artículos por año) fueron: Objetos de aprendizaje (5); Multimedia (5); Aprendizaje de Lenguas asistido por ordenador (5); Estilos de aprendizaje (4) y Adopción de tecnología (4). El tema «E-learning en Educación Superior» emerge en el año 2004 con 2 artículos (no hay ninguna publicación en el período 2000-2003) y crece hasta los 19 artículos en el año 2010.

Hsu et al. (2013) concluyen que, a menos que otra tecnología para el aprendizaje genere un cambio de paradigma, como lo hizo Internet, la dimensión «Aceptación/Actitud hacia tecnologías emergentes» no va a desarrollarse en el futuro como una línea de investigación relevante en Tecnología Educativa. Sin embargo, el uso pedagógico de las tecnologías y la eficacia de las estrategias de enseñanza para el logro de resultados de aprendizaje se prevé como una dimensión con una mayor producción científica. Para estos investigadores nos encontramos en la era de los «entornos de aprendizaje» con un gran interés de la TE en la interacción y la colaboración en contextos de enseñanza-aprendizaje basados en Internet.

No obstante, los miles de estudios en TE y meta-análisis de estas investigaciones han demostrado que la TE no ha alcanzado aún su supuesto potencial y, en los últimos años, el escepticismo sobre su eficacia se ha incrementado (Cuban, 2003). Uno de los factores más relevantes para explicar esta situación es el enfoque metodológico que se adopta para la realización de las investigaciones en TE. Frente a la defensa de los estudios experimentales (conocidos como RTCs o Randomized Controlled Trials) utilizados, por ejemplo, en la investigacion médica, que realizan determinados expertos en metodología de investigación educativa (v.gr. Slavin, 2002; Feuer, Towne, y Shavelson, 2002), otros reputados metodólogos, como Cronbach (1975) después de décadas de estudios experimentales, llegaron a la conclusión de que no podemos acumular generalizaciones a partir de numerosos estudios a pequeña escala para aplicar los resultados en aulas específicas en un tiempo determinado. "Cuando damos un peso apropiado a las condiciones locales, cualquier generalización es una hipótesis de trabajo, no una conclusión» (Cronbach, 1975, p. 125). Khun afirmó que no está claro que dispongamos aún de las «categorías conceptuales de investigación, herramientas de investigación y una selección apropiada de temas que nos conduzcan a comprender el proceso educativo. Existe una asunción general de que si tú tienes un problema relevante, la forma de resolverlo es aplicando la ciencia. Todo lo que debes hacer es llamar a las personas apropiadas y poner suficiente dinero para que en pocos años, lo hayas resuelto. Pero esto no funciona así, ni lo hará nunca» (cit. en Glass y Moore, 1989, p.1).

Sin embargo, los investigadores en educación parecemos ser incapaces de aprender de nuestra historia pasada y del insignificante impacto en la práctica educativa de nuestros estudios. Reeves (2006) considera que, al igual que en otros muchos contextos de la investigación educativa, la investigación en TE ha estado plagada de «diferencias no 
significativas» e incluso la mayoría de meta-análisis profundos de estudios de investigación cuasi-experimentales realizados por tecnológos educativos muestran resultados que son extremadamente limitados, en el mejor de los casos.

La revisión que Reeves (1995) llevó a cabo sobre cinco años de investigación en los artículos de las revistas Educational Technology Research y Development (ETRyD) y Journal of Computer-Based Instruction, evidenció que la mayoría de los estudios publicados contenían objetivos predictivos de hipótesis derivadas de teorías o de la comparación de un medio educativo con otro. A pesar de ser revistas con arbitraje, Reeves encontró que la mayoría de los estudios utilizaban diseños cuasi-experimentales erróneos y/o insuficientes medidas cuantitativas de variables relacionadas con el rendimiento o las actitudes. Como resultado de la naturaleza, generalmente pseudocientífica, de la mayoría de las publicaciones sobre TE, otros investigadores que realizaron meta-análisis, tuvieron que rechazar, con frecuencia, más del $75 \%$ de las publicaciones por una diversidad de errores de carácter estadístico, de carencia de bases teóricas que sustenten los estudios, inadecuada revisión de la literatura, implementación de tratamientos insuficientes, resultados de aprendizaje sin transcendencia o ilógicos para los investigadores, muestras no idóneas o discusión de resultados poco rigurosa. Otro ejemplo destacable es el meta-análisis que Bernard et al. (2004) llevaron a cabo sobre comparaciones empíricas de la formación a distancia frente a la formación presencial, durante el período 1985 y 2002. Aunque encontraron más de 1.000 estudios, muchos fueron desechados por no cumplir los criterios de selección. De la muestra final se extrajeron conclusiones con significatividad estadística, que demostraba una pequeña ventaja de la formación a distancia frente a la presencial. Aunque se trata de uno de los mejores estudios de esta naturaleza, sus resultados no ofrecían orientaciones específicas para los docentes, es decir, propuestas para la mejora de práctica educativa.

El tipo de investigación sobre comparación entre medios recogidos en la mayoría de los meta-análisis tiene una larga y dudosa historia en la TE. A pesar de las frecuentes recomendaciones en contra de este tipo de estudios, continúan siendo publicados con un pretexto u otro. El resultado más habitual es «no hay diferencias significativas en el aprendizaje» (Reeves, 2006).

De todo este panorama, se concluye que es evidente que existe una urgente necesidad de desarrollar un mejor enfoque para la investigación en TE. Esto supone que los tecnológos educativos que investigan en este ámbito deberían: (a) investigar problemas complejos en contextos reales en colaboración con docentes; (b) integrar principios de diseño instruccional conocidos e hipotéticos, con avances tecnológicos para obtener soluciones plausibles a estos problemas complejos y (c) llevar a cabo estudios rigurosos y reflexivos para examinar y refinar entornos innovadores de aprendizaje, así como definir nuevos principios educativos (Brown, 1992; Collins, 1992).

La conceptualización de la teoría educativa como independiente de la práctica, o como un «a priori» alejado de la experiencia real, debería ser redefinida por otra que reconozca que la construcción de la teoría educativa puede ser elaborada, de manera colaborativa, por investigadores y profesionales de la educación en contextos reales. El conocimiento del «diseño instruccional» no es un objeto que los investigadores «destilan» de experimentos para que luego sea aplicado por los docentes, como meros técnicos ejecutores de las decisiones de otros. Se trata de un conocimiento contextual, social y evolutivo. Por tanto, el objetivo esencial de la investigación en TE debería ser contribuir a resolver auténticos problemas educativos y proponer principios que puedan justificar futuras decisiones de implementación en los procesos de enseñanza-aprendizaje. Como sostiene Reeves (2006, pp. 61-62), «nuestra meta no debería ser desarrollar conocimientos teóricos esotéricos que creemos que los docentes deberían aplicar. Esto no ha funcionado durante los últimos 50 años, y no funcionará en el futuro.» 


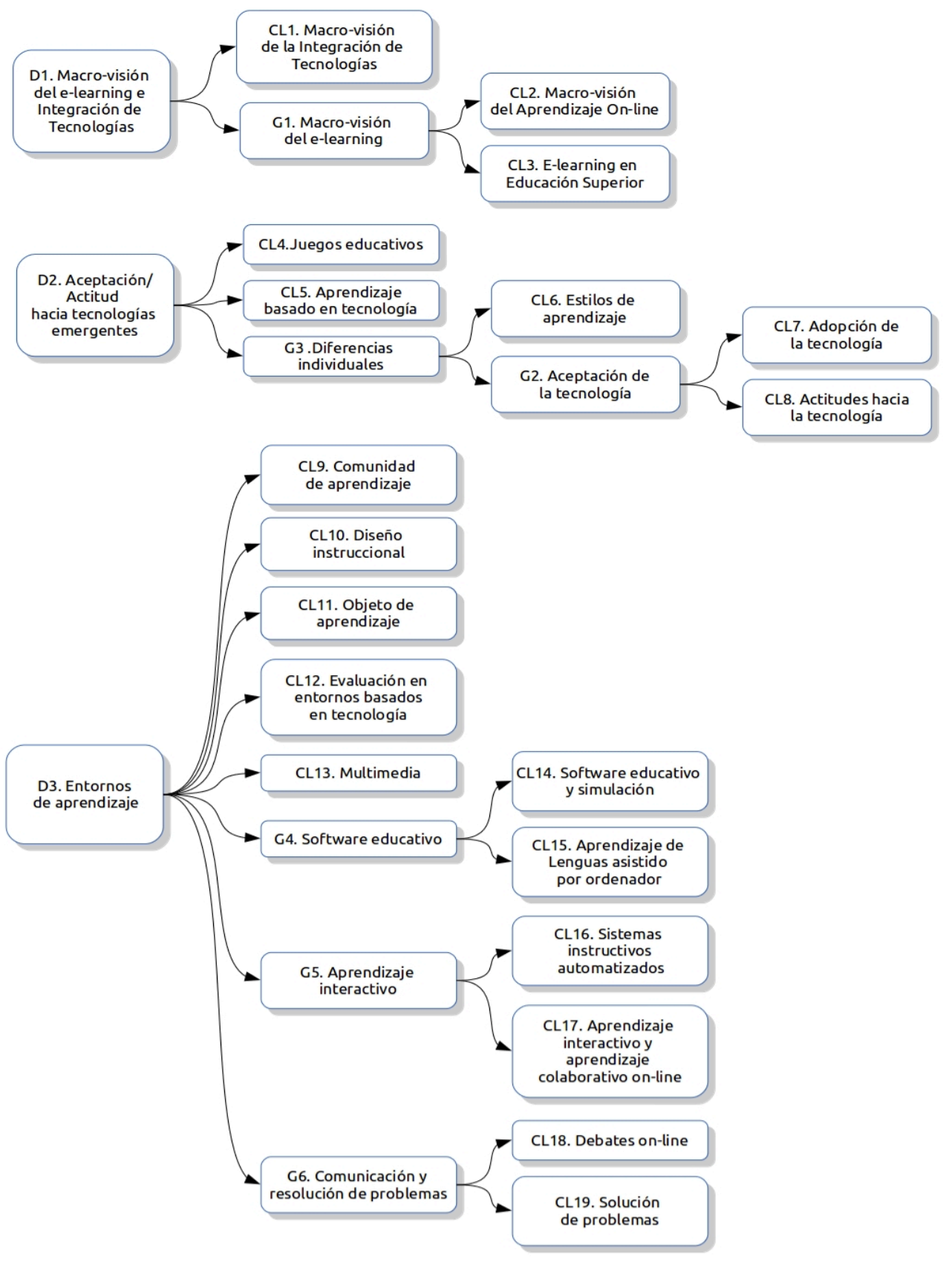

Figura 1. Árbol de cluster de los principales temas de investigación (trad. de Hsu et al. 2013, p. 693)

Los actuales entornos de aprendizaje más complejos, distribuidos, contectados y flexibles, exigen incorporar una nueva metáfora sobre la "creación del conocimiento», es decir, la creación colectiva del conocimiento por medio de objetos de actividad compartidos. Este enfoque se conoce como "aprendizaje trialógico» (co-evolución de investigadores, de objetos de investigación). Los objetos pueden ser conceptuales (preguntas, teorías, diseños), materiales (prototipos, productos concretos) o representar prácticas sobre las que reflexionar 0 transformar colectivamente. El aprendizaje es un proceso de indagación innovadora en el que el objetivo es refinar progresivamente los artefactos de conocimiento y desarrollar procesos a largo plazo para extender el conocimiento de la comunidad y sus competencias (Hakkarainen y Paavola, 2009). 
El avance del conocimiento y la creatividad pueden ser comprendidos como una «actividad trialógica», es decir, una actividad en la que las personas trabajando conjuntamente están elaborando un objeto compartido, ya sea un problema de investigación, una teoría, un plan, un diseño, un producto o una práctica (para ser reflexionada o transformada). Las seis características básicas del «aprendizaje trialógico» son (Hakkarainen, 2008): (1) orientación hacia objetos compartidos de actividad que son desarrollados colaborativamente; (2) búsqueda del avance del conocimiento en un proceso constante y a largo plazo; (3) los procesos de creación de conocimientos tienen lugar por medio de la interacción entre actividades individuales y colectivas; (4) Interrelación de prácticas de conocimiento entre comunidades educativas, profesionales y de investigación; (5) mediación tecnológica diseñada para la creación, construcción y compartición del conocimiento de modo colaborativo a largo plazo y (6) desarrollo a través de la transformación y la reflexión.

La investigación sobre el «aprendizaje triálogico» se ha desarrollado a través del proyecto KPL1 (Knowledge Practices Laboratory) que estudió la creación colaborativa de conocimiento en el ámbito de la Tecnología Educativa, a través del modelo Design-Based Research (en adelante DBR). Los principios para el "aprendizaje trialógico» que se extraen de esta investigación son analizados a través de una triple perspectiva: teoría, pedagogía y tecnología (Paavola, Lakkala, Muukkonen, Kosonen, y Karlgren, 2011).

Necesitamos reorientar la investigación en TE hacia un enfoque que contribuya a generar un impacto real y eficaz sobre la práctica educativa, es decir, una orientación maś responsable y comprometida socialmente. Si no conseguimos ofrecer una investigación educativa de mayor calidad, los docentes, gestores educativos y responsables políticos continuarán teniendo problemas para desarrollar una Educación Digital que contribuya a la necesaria reforma de la educación en todos sus niveles. Desde nuevas teorías sobre la generación del conocimiento (como el «aprendizaje trialógico») y enfoques metodológicos (como el DBR) se puede articular una fecunda línea de investigación en el ámbito de la TE.

\section{EL MODELO DBR (DESIGN-BASED RESEARCH) PARA LA INVESTIGACIÓN EN TECNOLOGÍA EDUCATIVA}

Brown (1992) fue la primera investigadora que desarrollo el modelo DBR. Desde entonces se han publicado diversos monográficos en revistas científicas de reconocido prestigio como Educational Researcher (2003, vol. 32, n. ${ }^{\circ} 1$ ), Journal of the Learning Sciencies (2004, vol. 13, n. ${ }^{\circ}$ 1) o Educational Psychologist (2004, vol. 39, n. $\left.{ }^{\circ} 4\right)$. Así como varios handbooks sobre este enfoque metodológico, entre los que destacan los textos coordinados por van den Akker et al. (1999), van den Akker (2006), Kelly et al. (2008) y Plomp y Nieveen (2010). Una de las investigadoras que más ha publicado en la última década ha sido Diane Ketelhut con relación a uno de los proyectos más relevantes en el uso de DBR denominado «River City» ${ }^{2}$. Sin embargo, los manuales sobre metodología de investigación educativa no suelen presentar y comentar el modelo DBR. Aunque la denominación más consensuada en la comunidad científica es «design-based research», no existe un acuerdo en el uso de un único término para DBR. Algunos autores utilizan otras denominaciones alternativas como «design research», «formative research» 0 «design experiments».

Cualquier investigación científica tiene como finalidad esencial alcanzar un saber más profundo y riguroso con el objetivo de contribuir al corpus de conocimientos de un área de investigación. Además, en el caso de la investigación educativa, se persigue aportar nuevas comprensiones de los fenómenos educativos emergentes y realizar propuestas para la mejora de la práctica, que puedan sustentar la toma de decisiones. Siguiendo a Stokes (1997), DBR es la formulación educativa del cuadrante más productivo de su modelo (ver fig. 2), que incluye tanto la generación de investigación «básica» como la producción de aplicaciones prácticas.

\footnotetext{
${ }^{1}$ Sitio web del proyecto: http://www.kp-lab.org/

${ }^{2}$ The River City Project. A Multi-User Virtual Environment for Learning Scientific Inquiry and 21st Century Skills . http://muve.gse.harvard.edu/rivercityproject/index.html
} 
Fig. 2. Cuadrante de Pasteur (Stokes, 1997, p. 73)

La investigación está inspirada por:

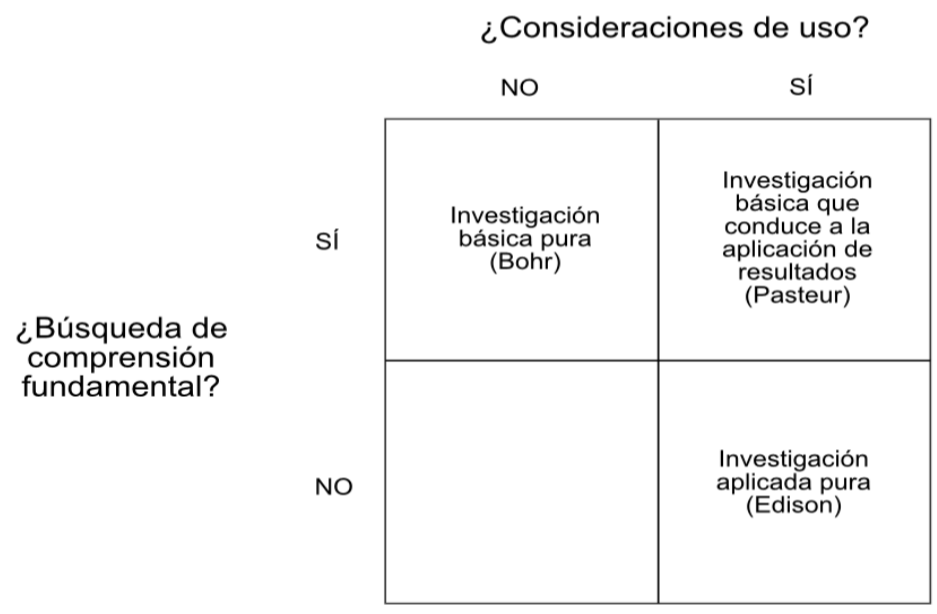

Entre las diversas funciones que cumple la investigación podemos identificar las siguientes, que ilustramos con un ejemplo de pregunta de investigación para cada tipología (Tabla 1).

Tabla 1. Funciones de la investigación educativa.

\begin{tabular}{lc}
\hline Tipología & Ejemplo de pregunta de investigación en TE \\
\hline Describir & $\begin{array}{c}\text { ¿Cuáles son las barreras que experimentan los docentes en la integración de las } \\
\text { tecnologías digitales en el currículo? }\end{array}$ \\
\hline Comparar & $\begin{array}{c}\text { ¿Cuáles son las diferencias y similitudes entre la consideración de la } \\
\text { competencia digital en los currículos de diferentes sistemas educativos? }\end{array}$ \\
\hline Evaluar & $\begin{array}{c}\text { ¿Cuáles son las fortalezas y debilidades de un proyecto o programa de } \\
\text { introducción de tecnologías digitales en las aulas (v.gr. Escuela 2.0)? }\end{array}$ \\
\hline Explicar o predecir & $\begin{array}{c}\text { ¿Cuáles son las causas del limitado uso de las tecnologías digitales en las } \\
\text { aulas? ¿Qué factores influyen en el uso frecuente de las tecnologías } \\
\text { digitales en las aulas? }\end{array}$ \\
\hline Diseñar o desarrollar & $\begin{array}{c}\text { ¿Cuáles son las características de un estrategia eficaz de utilización de las } \\
\text { tecnologías digitales para el logro de determinadas competencias o } \\
\text { resultados de aprendizaje? }\end{array}$ \\
\hline
\end{tabular}

El enfoque DBR se centra en función de «diseñar» o "desarrollar». Se define como un estudio sistemático de diseño, desarrollo y evaluación de intervenciones educativas (como programas, estrategias de enseñanza-aprendizaje, materiales, productos y/o sistemas) con el fin de aportar soluciones a problemas complejos de la práctica educativa y contribuir al avance del conocimiento pedagógico sobre las características de estas intervenciones y sus procesos de diseño y desarrollo (Plomp y Nieveen, 2010). Es un modelo metodológico que se orienta a la creación de nuevas teorías, artefactos y prácticas que tengan en cuenta problemas significativos e impacten en el aprendizaje y la enseñanza en contextos reales (Barab y Squire, 2004).

Por consiguiente, una de las características centrales de DBR es que se parte de un problema. Una idea reiterada en la literatura es que DBR se inicia con un problema que tiene significación científica y práctica. Por eso para examinar el progreso de DBR es preciso incluir el tipo de problemas abordados, las soluciones desarrolladas o las teorías elaboradas. Las preguntas que los investigadores se formulan son del tipo: "¿Qué alternativas hay a las actuales prácticas educativas?» o "¿Cómo pueden estas alternativas ser establecidas y sostenidas?» (Edelson, 2006). Como señala Kelly (2006), el DBR es un tipo de investigación exploratoria que destaca el valor de la novedad y apuesta por enfoques no-convencionales y 
creativos. No depende de estructuras previas y variables a medir, sino que intenta aportar soluciones para los problemas tal y como emergen.

La investigación que aplica DBR busca una influencia directa en la práctica docente y también contribuir a las decisiones sobre política educativa (Romero-Ariza, 2014). Su finalidad es ofrecer soluciones a problemas educativos complejos, a partir de los resultados de la investigación. Se orienta hacia aquellos fenómenos educativos que son necesarios conocer y comprender para aportar innovaciones que contribuyan a una mejora del proceso enseñanzaaprendizaje en contextos específicos. Muchos estudios publicados son capaces de demostrar un impacto potencial, pero no un impacto auténtico sobre la práctica. Una de las principales metas de DBR es generar teorías que puedan ser de valor para los profesionales de la educación (v.gr. Barab, Gresalfi, y Ingram-Goble, 2010; Clarke y Dede, 2009).

En consecuencia, el requisito de desarrollar principios prácticos es un elemento clave del DBR, frente a otro tipo de investigación educativa que unilateralmente examina determinadas variables en el aula y luego el investigador desaparece una vez que el experimento ha concluido. Estos principios prácticos no se diseñan para crear teorías descontextualizadas que funcionan con iguales efectos en todos los contextos. Más bien, estos principios reflejan las condiciones en los operan dentro del contexto (Anderson y Shattuck, 2012). "Las orientaciones filosóficas generales para temas educativos -como el constructivismo- son importantes para la práctica docente, pero a menudo fallan en proporcionar una orientación detallada en la organización del proceso enseñanza-aprendizaje» (Cobb, Confrey, diSessa, Lehrer, y Schauble, 2003, p. 10). En la actualidad es difícil encontrar una investigación educativa que los docentes identifiquen como influyente en su práctica educativa.

A partir de una revisión de la literatura científica, los investigadores colaboran con los docentes para diseñar y desarrollar intervenciones que se analizan sistemática y rigurosamente, en diferentes versiones (o prototipos) que son aplicadas en contextos reales y sobre los que se reflexiona para generar teorías y principios didácticos. Este modelo implica un grado de innovación suficiente para explorar un territorio que no puede ser explorado con medios tradicionales, pero debe estar guiado por una teoría sólida, para asegurar que no es meramente especulativo (Edelson, 2006).

El modelo DBR consta de una serie de estadios o fases (Plomp y Nieveen, 2010):

a) Investigación preliminar: necesidades y análisis del contexto, revisión de la literatura, desarrollo de un marco conceptual o teórico para el estudio.

b) Fase de prototipo: fase de diseño iterativo que consta de iteraciones, cada una de las cuales es un micro-ciclo de investigación, con evaluación formativa como la actividad de investigación más importante con el objetivo de mejorar y redefinir la intervención.

c) Fase de evaluación: evaluacion «semi-sumativa» para concluir si la solución o intervención satisface las especificaciones pre-determinadas. Como esta fase ofrece, a menudo, recomendaciones para la mejora de la intervención, se denomina «semisumativa».

Mediante las actividades desarrolladas en las diferentes fases, los investigadores realizan una reflexión sobre las evidencias encontradas y recogidas en una documentación sistemática del problema, para generar teorías o principios de diseño instruccional, que constituyen la producción científica del proyecto de investigación. De hecho, se puede afirmar que la doble tarea de reflexión y documentación sistemática convierten el diseño y desarrollo de la intervención educativa en DBR.

El modelo DBR no busca el estudio de variables aisladas, porque adopta un enfoque holísitico frente a la comprensión de la complejidad de los problemas educativos. Se orienta hacia objetos y procesos específicos en contextos concretos, pero estudian las intervenciones como fenómenos integrales y significativos de ese entorno educativo específico. Por lo tanto, la investigación educativa no conduce a realizar generalizaciones independientes del contexto, que son las que se emiten en los estudios experimentales realizados sobre una muestra de una 
población mediante el uso de determinados estadísticos. No podemos considerar que DBR sea una «nueva metodología de investigación» puesto que utiliza métodos de investigación mixtos (cuantitativos y cualitativos) ya existentes y sigue las normas, utiliza los procesos y técnicas propias de cada método (McKenney y Reeves, 2013).

En resumen, las características clave de DBR son las siguientes: (i) «intervencionista», es decir, una investigación orientada sobre el diseño de intervenciones en el contexto real de educación o formación. La intervención puede ser una actividad de aprendizaje, un tipo de evaluación, la introducción de una actividad administrativa (como un cambio en la organización), o una intervención tecnológica, por mencionar algunas de los tipos más comunes (Anderson y Shattuck, 2012); (ii) "orientada a procesos», es decir, combinada con esfuerzos para comprender (saber) y mejorar las intervenciones (saber hacer), y (iii) «orientada a la teoría», puesto que utiliza el estado del arte o revisión de la literatura, junto con el estudio de campo y la evaluación de los prototipos, para contribuir a la construcción de teorías (Plomp y Nieveen, 2010). La figura 2 ilustra las diferentes fases, componentes y proceso de una

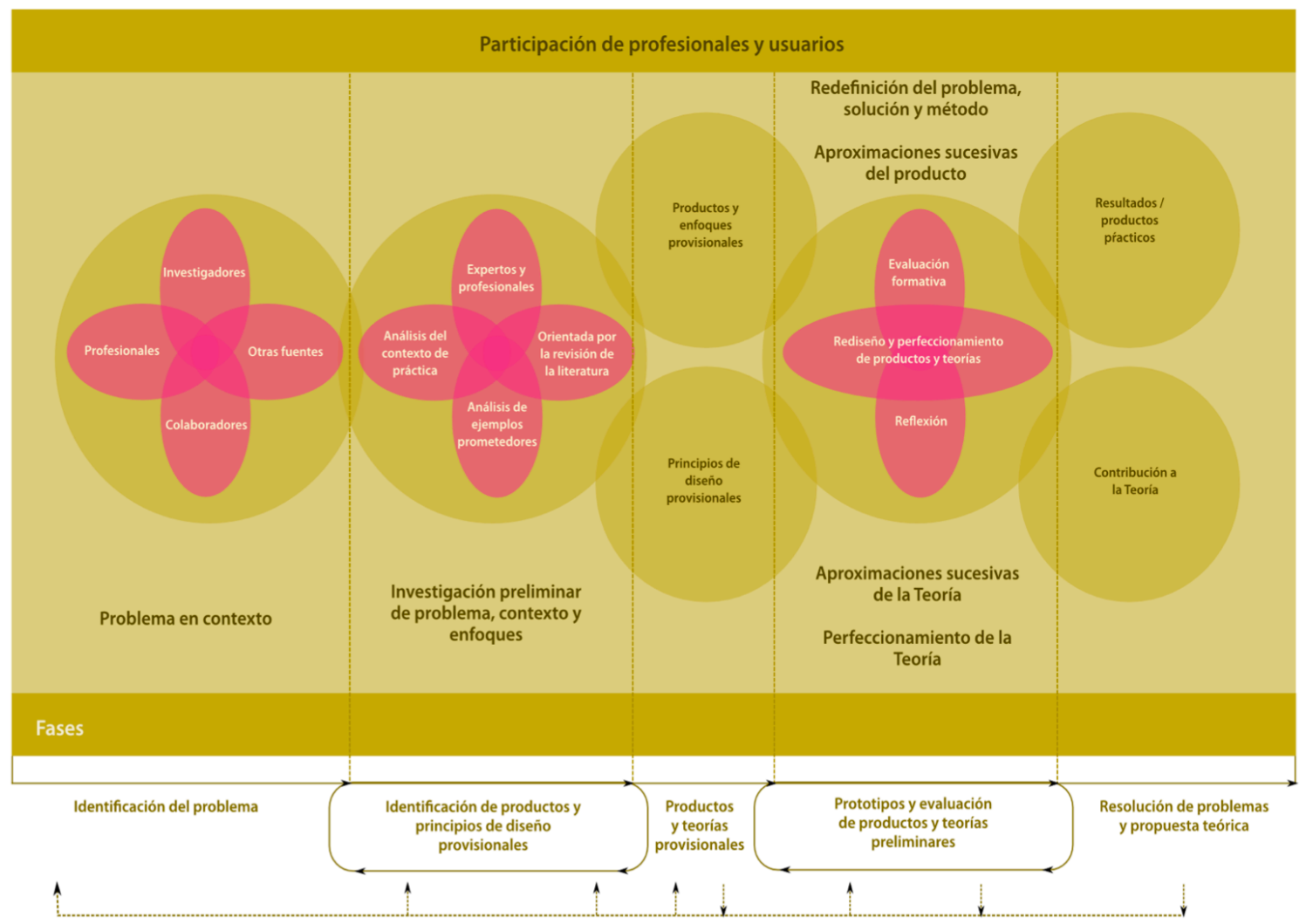

investigación según el modelo DBR.

Figura 2. Modelo genérico de Diseño de Investigación (Wademan, 2005) Elaboración propia trad. de Plomp y Nieveen (2010, p. 16)

Un estudio DBR se enmarca en un contexto educativo real para aportar validez a la investigación y asegurar que los resultados pueden ser utilizados eficazmente para informar, evaluar y mejorar la práctica, al menos, en el contexto donde se ha llevado a cabo dicho estudio. Por tanto, se orienta al diseño y análisis de una intervención significativa. La selección y creción de la intervención es una tarea colaborativa tanto de investigadores como de docentes. La creación comienza con una evaluación rigurosa del contexto local; se basa en una selección de la literatura más relevante y de la teoría y práctica de otros contextos; y está diseñada específicamente para superar algún problema o crear una mejora en la práctica local. La clave de la calidad de los resultados del proyecto de investigación está en el diseño de estas intervenciones. El equipo de investigación debe documentar rigurosamente todo el proceso de diseño y desarrollo de la intervención. Los datos obtenidos permiten elaborar informes que puedan ser utilizados, no solo por los docentes del estudio, sino en otros contextos educativos, si se juzga que podrían obtenerse logros similares (Anderson y Shattuck, 2012). 
La práctica de diseñar exige habitualmente la creación y prueba de «prototipos», que se ponen en práctica en situaciones reales y se van mejorando paulatinamente en una evolución constante. Cualquier intervención no produce todos los efectos deseados, es siempre perfectible y debe someterse a evaluación. En DBR la «iteración múltiple» es uno de sus desafíos más importantes por su dificultad en la aplicación y por la indefinición sobre sus límites temporales.

Otra característica básica en un estudio DBR es la colaboración entre investigadores y profesionales de la educación. Son roles diferenciados, pero cuya cooperación es imprescindible para el diseño y desarrollo de la investigación. Por una parte, se reconoce que el docente está muy ocupado con las actividades propias de su práctica educativa y que, por lo general, carece de una formación especializada para la implementación de una investigación rigurosa. Por otra parte, el investigador desconoce, con frecuencia, las complejidades del sistema educativo y de la realidad de las instituciones escolares y, en consecuencia, no tiene la capacidad por sí solo para identificar problemas, diseñar y valorar el impacto de las intervenciones. Por tanto, el acuerdo de colaboración incluye negociar deste la identificación del problema inicial, a través de la revisión de la literatura, al diseño y desarrollo de la intervención, evaluación y publicación de los principios teóricos y prácticos (Anderson y Shattuck, 2012).

Se definen cuatro pasos para hacer que un estudio DBR sea un proceso útil para el desarrollo de la teoría educativa (Edelson, 2006): (1) Las decisiones deben estar basadas en una combinación de la investigación previa y las teorías emergentes del diseñador. (2) El proceso debe ser profunda y sistemáticamente documentado. (3) Una evaluación formativa para identificar debilidades en el análisis del problema, el diseño de la solución o del procedimiento. (4) La generalización que permita ofrecer teorías de dominio, estructuras de diseño o metodologías de diseño. Las teorías de dominio se refieren a generalización de una parte de un análisis de problema. Hay dos tipos de teorías: "Teoría de contexto», por ejemplo, la descripción de las necesidades de una determinada población de estudiantes, la organización de una institución educativa o la naturaleza de determinada asignatura; y «Teoría de resultados» que describe los efectos de las interacciones entre los componentes de un diseño. Explica por qué un diseñador podría escoger ciertos elementos para un diseño en un contexto y otros elementos en otros. Las «estructuras de diseño» son soluciones que proporcionan orientaciones para lograr determinadas metas en un contexto particular y las «metodologías de diseño» describen un procedimiento general que une las metas y contextos en un conjunto apropiado de procesos.

\subsection{Relevancia del modelo DBR en la investigación sobre Tecnología Educativa: algunos ejemplos}

Algunos estudios de revisión de la evolución del modelo DBR han comprobado un elevando porcentaje de estudios (70\%) vinculados directamente con el uso de las tecnologías digitales (Anderson y Shattuck, 2012). Una revisión no sistemática de la producción científica en los últimos años nos muestra que DBR es un modelo vigente e idóneo en la investigación sobre TE. Esta selección no exhaustiva incluye algunos estudios que ejemplifican la potencialidad del DBR para el desarrollo de una investigación educativa que aspire a ser algo más que un mero ejercicio academicista y busque la influencia directa sobre la práctica docente y la toma de decisiones en la política educativa.

Aunque el primer caso no identifica explícitamente el uso de DBR, bien podría ser considerado un tipo de estudio que ha desarrollado los principios y procedimientos de este enfoque metodológico. El proyecto ALTER «Alternativas Telemáticas en Aulas Hospitalarias: una experiencia educativa» (Serrano Sánchez y Prendes Espinosa, 2015, 2014) es un estudio que trata de resolver el problema de cómo las tecnologías pueden mejorar los procesos educativos en un hospital y las premisas a tener en cuenta. Se realiza una propuesta de integración curricular de TIC válida para los maestros del hospital, que aporta un conocimiento útil para resolver las necesidades de un contexto educativo real. 
El estudio de Sáez-López et al. (2016) utiliza DBR como opción metodológica para estudiar los efectos de la introducción del pensamiento computacional a través de la programación visual (Scratch) en cinco centros de Educación Primaria con alumnos de $5^{\circ}$ y $6^{\circ}$ curso $(\mathrm{N}=107)$. La intervención tuvo lugar durante dos cursos académicos y se diseñó para su uso en las áreas de Ciencias y Sociales. Como resultados con aplicación directa en el contexto educativo analizado se comprobó la relevancia de un enfoque pedagógico activo para la adquisición de competencias digitales y la motivación que generó en los estudiantes el uso de la programación visual dentro de una metodología PBL (Project Based Learning) para la adquisición de aprendizajes específicos.

El propósito de la investigación de Wang et al. (2014) es promover en maestros de Educación Primaria especializados en Ciencias $(\mathrm{N}=25)$ el uso de las tecnologías digitales para modificar sus prácticas tradicionales por otras centradas en el estudiante y con orientación constructivista. El estudio DBR se desarrolló durante un período de cuatro años e implicó a 24 centros escolares. Los resultados revelaron un cambio en la metodología docente que permitió a los estudiantes mayor autonomía en el uso de las tecnologías digitales y se comprobó un impacto positivo en las competencias digitales y los aprendizajes en Ciencias.

Por último, en el ámbito de la formación online, el estudio de Shattuck y Anderson (2013) utilizaron el modelo DBR para evaluar un curso de formación de profesorado diseñado como una posible solución al problema que tienen algunas universidades para proporcionar una formación de calidad y accesible a docentes que están haciendo una transición hacia la enseñanza online. El estudio evaluó si el contenido, estructura y enfoque pedagógico del curso ayudaba eficazmente a la formación de docentes online. Como resultado se aportan una serie de principios prácticos que se consideran transferibles a diferentes contextos universitarios.

\section{CONCLUSIONES}

En resumen se pueden identificar tres motivos por los que este enfoque metodológico es idóneo en la investigación sobre TE (van den Akker, Branch, Gustafson, Nieveen, y Plomp, 1999): (a) Incrementa la relevancia de la investigación para la práctica y la política educativa. Se ha criticado el débil vínculo entre la investigación educativa en TE y la práctica docente. Si se tiene éxito en generar descubrimientos que son percibidos más ampliamente como relevantes y significativos, las oportunidades para la mejora de la política educativa en todos sus niveles se verán incrementadas. (b) Desarrolla teorías fundamentadas empíricamente a través de estudios tanto del proceso de aprendizaje como de los medios (tecnologías) que apoyan ese proceso. (c) Incrementa la solidez del diseño. Muchos diseñadores educativos se orientan hacia la construcción de soluciones innovadoras sobre problemas educativos emergentes, pero su comprensión, a menudo, permanece implícita en las decisiones tomadas y los resultados del diseño. Desde esta perspectiva, existe la necesidad de extraer más aprendizaje explícito que pueda avanzar en posteriores diseños.

\section{RECONOCIMIENTOS}

Este artículo es resultado del proyecto de investigación «Ecologías del aprendizaje en contextos múltiples: análisis de proyectos de educación expandida y conformación de ciudadanía», financiado por el Ministerio de Economía y Competitividad - Proyectos I+D, del Programa Estatal de Fomento de la Investigación científica y técnica de excelencia, Subprograma Estatal de Generación del Conocimiento. Referencia: EDU2014-51961-P.

\section{REFERENCIAS BIBLIOGRÁFICAS}

Anderson, T., y Shattuck, J. (2012). Design-Based Research: A Decade of Progress in Education Research? Educational Researcher, 41(1), 16-25. http://doi.org/10.3102/0013189X11428813

Barab, S. A., Gresalfi, M., y Ingram-Goble, A. (2010). Transformational Play Using Games to Position Person, Content, and Context. Educational Researcher, 39(7), 525-536. http://doi.org/10.3102/0013189X10386593 
Barab, S.A., y Squire, K. (2004). Introduction: Design-Based Research: Putting a Stake in the Ground. The Journal of the Learning Sciences, 13(1), 1-14.

Baydas, O., Kucuk, S., Yilmaz, R. M., Aydemir, M., y Goktas, Y. (2015). Educational technology research trends from 2002 to 2014. Scientometrics, 105(1), 709-725. http://doi.org/10.1007/s11192-015-1693-4

Bernard, R. M., Abrami, P. C., Lou, Y., Borokhovski, E., Wade, A., Wozney, L., ... Huang, B. (2004). How Does Distance Education Compare With Classroom Instruction? A MetaAnalysis of the Empirical Literature. Review of Educational Research, 74(3), 379-439. http://doi.org/10.3102/00346543074003379

Brown, A. L. (1992). Design Experiments: Theoretical and Methodological Challenges in Creating Complex Interventions in Classroom Settings. The Journal of the Learning Sciences, 2(2), 141-178.

Clarke, J., y Dede, C. (2009). Design for Scalability: A Case Study of the River City Curriculum. Journal of Science Education and Technology, 18(4), 353-365. http://doi.org/10.1007/s10956-009-9156-4

Cobb, P., Confrey, J., diSessa, A., Lehrer, R., y Schauble, L. (2003). Design Experiments in $\begin{array}{llll}\text { Educational Research. } \quad \text { Educational } & \text { Researcher, }\end{array}$ http://doi.org/10.3102/0013189X032001009

Collins, A. (1992). Toward a Design Science of Education. En E. Scanlon y T. O'Shea (Eds.), New Directions in Educational Technology (pp. 15-22). Berlin: Springer.

Cuban, L. (2003). Oversold and underused: computers in the classroom. Cambridge, Massachusetts: Harvard University Press.

Cronbach, L. J. (1975). Beyond the two disciplines of scientific psychology. American Psychologist, 30(2), 116-127. http://doi.org/10.1037/h0076829

Edelson, D. C. (2006). Balancing innovation and risk. En J. J. H. van den Akker (Ed.), Educational design research (pp. 100-106). London: Routledge.

Feuer, M. J., Towne, L., y Shavelson, R. J. (2002). Scientific Culture and Educational Research. Educational Researcher, 31(8), 4-14. http://doi.org/10.3102/0013189X031008004

Glass, G. V., y Moore, N. (1989). Research and practice: Universities and schools. Presentado en Second Annual Meeting of the Arizona Educational Research Organization. Recuperado a partir de http://www.gvglass.info/papers/research-practice.pdf

Hakkarainen, K. (2008). Toward a trialogical approach to learning: Personal reflections. LLineLifelong Learning in Europe, (13), 22-29.

Hakkarainen, K., y Paavola, S. (2009). Toward a trialogical approach to learning. En B. Schwarz, T. Dreyfus, y R. Hershkowitz (Eds.), Transformation of knowledge through classroom interaction (pp. 65-80). London; New York: Routledge.

Hsu, Y.-C., Hung, J.-L., y Ching, Y.-H. (2013). Trends of educational technology research: more than a decade of international research in six SSCl-indexed refereed journals. Educational Technology Research and Development, 61(4), 685-705. http://doi.org/10.1007/s11423-0139290-9

Kelly, A. E. (2006). Quality criteria for design research. Evidences and commitments. En J. J. H. van den Akker (Ed.), Educational design research (pp. 107-118). London: Routledge. 
Kelly, A. E., Lesh, R. A., y Baek, J. Y. (Eds.). (2008). Handbook of design research methods in education: innovations in science, technology, engineering, and mathematics learning and teaching. New York: London: Routledge.

McKenney, S., y Reeves, T. C. (2013). Systematic Review of Design-Based Research Progress: Is a Little Knowledge a Dangerous Thing? Educational Researcher, 42(2), 97-100. http://doi.org/10.3102/0013189X12463781

Paavola, S., Lakkala, M., Muukkonen, H., Kosonen, K., y Karlgren, K. (2011). The roles and uses of design principles for developing the trialogical approach on learning. $A L T-J$ : Research in $\quad$ Learning 233-246. http://doi.org/10.1080/21567069.2011.624171

Plomp, T., y Nieveen, N. M. (2010). An introduction to educational design research. Enschede: SLO - Netherlands Institute for Curriculum Development. Recuperado a partir de http://www.slo.nl/downloads/2009/Introduction_20to_20education_20design_20research.pdf

Romero-Ariza, M. (2014). Uniendo investigación, política y práctica educativas: DBR, desafíos y oportunidades. Magis. Revista Internacional de Investigación en Educación, 7(14), 159. http://doi.org/10.11144/Javeriana.M7-14.UIPP

Reeves, T. C. (1995). Questioning the Questions of Instructional Technology Research. Presentado en Annual National Convention of the Association for Educational Communications and Technology (AECT). Recuperado a partir de http://eric.ed.gov/?id=ED383331

Reeves, T. C. (2006). Design research from a technology perspective. En J. J. H. van den Akker (Ed.), Educational design research (pp. 52-66). London: Routledge.

Sáez-López, J.-M., Román-González, M., y Vázquez-Cano, E. (2016). Visual programming languages integrated across the curriculum in elementary school: A two year case study using «Scratch» in five schools. Computers y Education, 97, 129-141. http://doi.org/10.1016/j.compedu.2016.03.003

Serrano Sánchez, J. L., y Prendes Espinosa, M. P. (2015). Integración de TIC en aulas hospitalarias como recursos para la mejora de los procesos educativos. ESE: Estudios sobre educación, (28), 187-210. http://doi.org/10.15581/004.28.187-210

Serrano Sánchez, J. L., y Prendes Espinosa, M. P. (2014). TIC para la mejora educativa en aulas hospitalarias. Pixel-Bit: Revista de medios y educación, (45), 23-36. http://doi.org/http://dx.doi.org/10.12795/pixelbit.2014.i45.02

Shattuck, J., y Anderson, T. (2013). Using a design-based research study to identify principles for training instructors to teach online. The International Review of Research in Open and Distributed Learning, 14(5). Recuperado a partir de http://www.irrodl.org/index.php/irrodl/article/view/1626

Slavin, R. E. (2002). Evidence-Based Education Policies: Transforming Educational Practice and Research. Educational Researcher, 31(7), 15-21. http://doi.org/10.3102/0013189X031007015

Van den Akker, J.J.H., Branch, R. M., Gustafson, K., Nieveen, N., y Plomp, T. (1999). Design Approaches and Tools in Education and Training. Dordrecht: Springer. http://dx.doi.org/10.1007/978-94-011-4255-7

Van den Akker, J. J. H. (Ed.). (2006). Educational design research. London: Routledge.

Wang, S.-K., Hsu, H.-Y., Reeves, T. C., y Coster, D. C. (2014). Professional development to enhance teachers' practices in using information and communication technologies (ICTs) as 
cognitive tools: Lessons learned from a design-based research study. Computers $y$ Education, 79, 101-115. http://doi.org/10.1016/j.compedu.2014.07.006

\section{INFORMACIÓN SOBRE EL AUTOR}

\section{Jesús Valverde Berrocoso \\ Universidad de Extramadura}

Doctor en Pedagogía por la Universidad de Salamanca y profesor titular de Universidad en el Departamento de Ciencias de la Educación de la Facultad de Formación del Profesorado de la Universidad de Extremadura (Cáceres - España). Director del Campus Virtual de la Universidad de Extremadura (CVUEX) y Director del Campus Virtual Compartido del Grupo G9 de Universidades (CVC-G9). Director de la Revista Latinoamericana de Tecnología Educativa (RELATEC). Miembro de la Red Universitaria de Tecnología Educativa (RUTE) y de la Red Universitaria de Investigación e Innovación Educativa (REUNI+D). Coordino el grupo de investigación "Nodo Educativo» especializado en Tecnología Educativa cuyas principales líneas de investigación son las siguientes: 'Educación Digital en contextos de enseñanzaaprendizaje', 'Entornos virtuales de enseñanza-aprendizaje en Educación Superior', 'Diseño y elaboración de materiales didácticos digitales' y 'Videojuegos y Educación'.

Web personal: http://jesusvalverde.es/

Máster en Educación Digital: http://mastereducaciondigital.unex.es/

Nodo Observa: http://www.nodo-observa.es/

Twitter: @jesus_valverde / https://twitter.com/jesus_valverde

\section{$(\mathrm{Cc}) \mathrm{EY}-\mathrm{NO}$}

Los textos publicados en esta revista están sujetos a una licencia de Reconocimiento 4.0 España de Creative Commons. Puede copiarlos, distribuirlos, comunicarlos públicamente y hacer obras derivadas siempre que reconozca los créditos de las obras (autoría, nombre de la revista, institución editora) de la manera especificada por los autores o por la revista. La licencia completa se puede consultar en:Licencia Creative Commons Atribución-NoComercial 4.0 Internacional. 